\title{
Phase-matching conditions for nonlinear frequency conversion by use of aligned molecular gases
}

\author{
R. A. Bartels, N. L. Wagner, M. D. Baertschy, J. Wyss, M. M. Murnane, and H. C. Kapteyn \\ JILA and the Department of Physics, University of Colorado, Boulder, Colorado 80309-0440
}

Received August 15, 2002

\begin{abstract}
Transient birefringence can be induced in a gas of anisotropic molecules by an intense polarized laser pulse. We propose to use this birefringence to phase match nonlinear optical frequency-conversion processes. The conditions for anisotropic phase matching are derived, and experimental conditions required for phase-matched third-harmonic generation in a gas-filled hollow-core fiber are presented. We show that these conditions are experimentally feasible over a significant parameter range, making possible a new type of nonlinear optics. (C) 2003 Optical Society of America

OCIS codes: 190.0190, 190.2620.
\end{abstract}

The development of nonlinear optics ${ }^{1,2}$ has dramatically expanded the utility of laser sources, making it possible to coherently convert radiation from visible or near-infrared lasers into the terahertz ${ }^{3}$ visible, ${ }^{4}$ and extreme-ultraviolet regions of the spectrum. ${ }^{5-7}$ A key issue in nonlinear optics is phase matching of the conversion process, i.e., to create a situation in which the propagation velocity of the pump and signal fields is the same, allowing the signal to accumulate constructively over extended propagation.

The most common implementation of phase-matched frequency conversion is birefringent frequency doubling, in which an oriented anisotropic crystal allows the pump and signal waves to propagate with different polarizations but the same phase velocity. Since gases and noncrystalline materials normally exhibit neither birefringence nor second-order nonlinearities, this process has been demonstrated only using anisotropic crystals. However, gases are transparent over a much broader range of frequencies than solids, making them an attractive candidate for vacuum ultraviolet frequency conversion. Existing gas-phase frequency conversion techniques rely on either resonances $^{8,9}$ or waveguide propagation, ${ }^{7,10}$ but each has limitations. New techniques are needed to generate light over a broader range of frequencies and to support shorter-duration pulses.

Here, we propose a new phase-matched frequencyconversion technique for gaseous media: transient birefringent phase matching. In a molecular gas the intrinsic anisotropy in polarizability of the individual molecules normally is not observed, since the molecules are randomly oriented. However, a transient alignment can be imposed on the gas by use of an intense light field that induces a torque on any molecule that is not oriented either parallel or perpendicular to the laser field. ${ }^{11}$ For pulses shorter than the rotational period of the molecule, the induced torque is impulsive and results in a change in the rotational angular momentum and an excited distribution of rotational energy levels. The ensemble of molecules experiences a periodic realignment determined by the excited angular momentum states and the molecular rotational energy level structure. These alignments modify the macroscopic polarizability of the ensemble of molecules and induce a transient birefringence. Recently, we demonstrated that this time-dependent index can be used to spectrally broaden an ultrafast optical pulse with a negative chirp that is time compressed with a transparent window. ${ }^{12}$

In this Letter we calculate the conditions under which the birefringence created by an ensemble of aligned molecules can be used to phase match nonlinear frequency conversion. In particular, we calculate the conditions required to phase match third-harmonic generation in a hollow-core fiber, but these results can also be generalized to other nonlinear processes and interaction geometries.

The polarizability tensor $\overline{\overline{\boldsymbol{\alpha}}}_{m}$ of an isolated linear, axially symmetric molecule (e.g., $\mathrm{CO}_{2}, \mathrm{~N}_{2}$ ) is a diagonal second-rank tensor with elements $\left\{\alpha_{x^{\prime} x^{\prime}}, \alpha_{y^{\prime} y^{\prime}}, \alpha_{z^{\prime} z^{\prime}}\right\}=\left\{\alpha_{\perp}, \alpha_{\perp}, \alpha_{\|}\right\}$, where $\|$is the direction along the long axis of the molecule, $\perp$ is perpendicular to the long axis, and the primed coordinates are along the principal directions of the molecule. If all these molecules are aligned along the same direction, the macroscopic polarizability of the ensemble of molecules will be dictated by the polarizability properties of a single molecule, and the gas behaves as a positive uniaxial crystal. More generally, the molecules are not completely aligned. To calculate the macroscopic polarizability of the entire ensemble, we transform the linear polarizability tensor through the rotational degrees of freedom, $\overline{\overline{\boldsymbol{\alpha}}}_{m}=\overline{\overline{\boldsymbol{\alpha}}}_{m: \phi, \theta, \psi}$. The macroscopic polarizability, $\overline{\overline{\boldsymbol{\alpha}}}$, is given by the average of the ensemble of molecules over the rotational degrees of freedom, $\overline{\overline{\boldsymbol{\alpha}}}=\left\langle\left\langle\left\langle\overline{\overline{\boldsymbol{\alpha}}}_{m: \phi, \theta, \psi}\right\rangle_{\phi}\right\rangle_{\theta}\right\rangle_{\psi}$. The transformation used is a standard Euler transform, where $\phi$ and $\psi$ are rotational degrees of freedom that are unaffected by the alignment field and $\theta$ is the rotation angle from the alignment polarization direction. The average over the unaligned rotational degrees of freedom is defined as $\left\langle\overline{\boldsymbol{\alpha}}_{m: \phi, \theta, \psi}\right\rangle_{\phi, \psi}=1 / 2 \pi \int_{0}^{2 \pi}\left\langle\overline{\boldsymbol{\alpha}}_{m: \phi, \theta, \psi}\right\rangle_{\phi, \psi} \mathrm{d} \phi, \mathrm{d} \psi$, and the average over $\theta$ is determined by the degree of alignment imposed by the alignment pulse.

The aligned linear polarizability tensor is diagonal in the coordinates defined by the polarization direction of the alignment pulse. The polarization 
of the pump pulse defines the extraordinary axis of the aligned quasi-crystal, with a polarizability given by $\alpha_{z z}=\alpha_{\perp}+\Delta \alpha\left\langle\cos ^{2} \theta\right\rangle$. The polarizability of the directions perpendicular to this axis corresponds to $\alpha_{x x}=\alpha_{y y}=1 / 2\left(\alpha_{\perp}+\alpha_{\|}-\Delta \alpha\left\langle\cos ^{2} \theta\right\rangle\right)$ and defines the ordinary index of the quasi-crystal; the inset of Fig. 1 shows the geometry. Here, $\Delta \alpha=\alpha_{\|}-\alpha_{\perp}$ is the molecular polarizability anisotropy.

We can measure the components of the linear polarizability tensor of the aligned ensemble by recording the frequency shift imposed on a probe pulse by the temporal index of refraction variation of a revival. ${ }^{12}$ This frequency shift of $\Delta v(t)=$ $-1 / 2 \pi[\mathrm{d} \tilde{\varphi}(t) / \mathrm{d} t]$, where $t$ is the delay between the pump and probe pulses, is integrated to yield the revival phase and thus the instantaneous index of both polarizations. Figure 1 shows a measurement of the index as a function of time for the ordinary and extraordinary polarization for the first partial revival in $\mathrm{CO}_{2}$ gas and verifies the predicted ordinary and extraordinary indices imposed by the molecular alignment.

This calculation and measurement demonstrates the uniaxial nature of the aligned-molecule quasi-crystal. Moreover, we see that the gas is a positive uniaxial crystal at times when the molecules exhibit a net alignment $\left(\left\langle\cos ^{2} \theta\right\rangle>1 / 3\right)$ and a negative uniaxial in the case of a net antialignment $\left(\left\langle\cos ^{2} \theta\right\rangle<1 / 3\right)$. This polarizability can be translated into an index of refraction by use of the approximate expression $n_{i j}-1 \approx$ $\alpha_{i j}\left(p N_{\text {Torr }} / 2 \epsilon_{0}\right) \equiv p \delta n_{i j}$, where $p$ is the gas pressure in Torr, $N_{\text {Torr }}$ is the number density of molecules at 1-Torr pressure, $\epsilon_{0}$ is the dielectric permittivity, and $\delta n_{i j}$ is a convenient grouping of constants.

Given the induced birefringence, it is possible to determine phase-matching conditions in an aligned molecular gas. However, it is also necessary to consider the properties of the nonlinear susceptibility in the aligned frame. Here, we consider the simplest case of third-harmonic generation (THG). Thus, we need to consider the properties of the ensembleaveraged third-order nonlinear tensor and to determine which tensor elements are nonzero and can connect the fundamental and harmonic polarizations to make THG possible. This calculation follows the procedure outlined for the linear susceptibility; however, the third-order polarizability $\left[\overline{\overline{\boldsymbol{\chi}}}^{(3)}\right]$ is a fourthrank tensor and transforms with the relation $\chi_{i j k l}^{(3)}=$ $r_{i a} r_{j b} r_{k c} r_{j d} \chi_{a b c d}^{(3)}$, where $r_{n m}$ are direction cosines defined by the transformation from the aligned reference frame.

After evaluating the $\overline{\overline{\boldsymbol{\chi}}}^{(3)}$ tensor components of the aligned ensemble of molecules, we find that the tensor elements depend on the alignment of the molecular gas and the nonzero tensor components allow for two types of anisotropic phase matching. The first phase-matching condition requires the third harmonic to be polarized along the extraordinary $(z)$ polarization direction $\left(3 \omega_{0} \rightarrow z\right)$ and the fundamental along both the ordinary $(y)$ and the extraordinary $(z)$ axes $\left(\omega_{0} \rightarrow\right.$ $y, y, z)$, whereas the other nonzero tensor elements allow phase matching with the polarizations $\left(3 \omega_{0} \rightarrow y\right)$ and $\left(\omega_{0} \rightarrow y, z, z\right)$. We define these phase-matching conditions as type II $\alpha$ and type II $\beta$, respectively.

Because the hollow-core fiber geometry allows for an extended interaction length in a simplified, effective one-dimensional geometry, this is the case that we consider here. Since the optic axis of the aligned quasi-crystal is coincident with the aligning pump pulse polarization, a probe pulse that is traveling collinearly with the aligning pump pulse propagates orthogonally to the optic axis, resulting in the so-called noncritical phase-matching geometry.

The $k$ vector of a light wave propagating in a hollowcore fiber filled with an anisotropic gas can be written as $k_{i j} \approx 2 \pi / \lambda\left[1+p\left\langle\delta n_{i j}\right\rangle_{x y}-1 / 2\left(u_{n m} \lambda / 2 \pi a\right)^{2}\right]$, where $\lambda$ is the free-space wavelength, $u_{n m}$ is a mode constant, $p$ is the gas pressure, $a$ is the hollow-fiber radius, $\left\langle\delta n_{i j}\right\rangle_{x y}=\int \delta n_{i j}(x, y) I(x, y) \mathrm{d} x \mathrm{~d} y / \int I(x, y) \mathrm{d} x \mathrm{~d} y$ is the modal averaged index, and $I(x, y)$ is the spatial mode in the hollow-core fiber.

Under conditions of negligible pump depletion, the field of the $q$ th-harmonic signal generated in a nonlinear medium can be written as $E_{q}(\omega) \propto \int_{0}^{L} E^{q}(\omega) \exp (i \Delta k z) \mathrm{d} z$, where the phase mismatch is defined as $\Delta k=k(q \omega)-\sum_{j=1}^{q} k_{i}(\omega)$. For optimal signal conversion, the phase mismatch is zero, corresponding to an equalization of the phase velocity of the fundamental and harmonic fields. When $\Delta k=0$, we can relate the optimum pressure, $p_{\text {opt }}$, to the required alignment $\operatorname{cosine}\left\langle\cos ^{2} \theta\right\rangle$ by the expression $P_{0}=p_{\text {opt }}\left(\left\langle\cos ^{2} \theta\right\rangle-K\right)$, where the waveguide pressure, $P_{0}$, and the critical alignment, $K$, are determined by the characteristics of the molecular gas, the waveguide, and the type of phase matching. For type II $\alpha$ phase matching with all fields propagating in the fundamental mode, we find that $P_{0}=-4 /\left(9 \delta \Delta n^{3 \omega}\right)\left(\lambda u_{11} / 2 \pi a\right)^{2}$ and $K=\left(\delta n_{\|}^{\omega}+2 \delta n_{\perp}^{\omega}-3 \delta n_{\perp}^{3 \omega}\right) /\left(3 \delta \Delta n^{3 \omega}\right)$, whereas for type II $\beta$ phase matching $P_{0}=8 /\left[9\left(\delta \Delta n^{3 \omega}+\delta \Delta n^{\omega}\right)\right] \times$ $\left(\lambda u_{11} / 2 \pi a\right)^{2}$ and $K=\left(\delta n_{\perp}^{3 \omega}+\delta n_{\|}^{3 \omega}-5 / 3 \delta n_{\perp}^{3 \omega}-\right.$ $\left.1 / 3 \delta n_{\|}^{\omega}\right) /\left(\delta \Delta n^{3 \omega}+\delta \Delta n^{\omega}\right)$. We note that $K$ is the degree of alignment required by the molecular gas for phase matching of the nonlinear interaction in free space when the alignment, fundamental, and harmonic pulses propagate collinearly. At high optimal

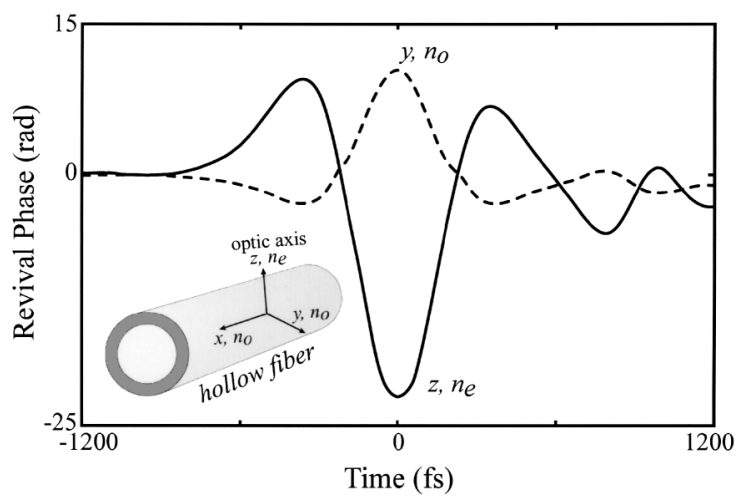

Fig. 1. Measured phase of the first rotational revival in $\mathrm{CO}_{2}$ for a probe polarized parallel (solid curve) and perpendicularly (dashed curve) to the alignment pulse polarization direction. The inset shows the alignment geometry. 

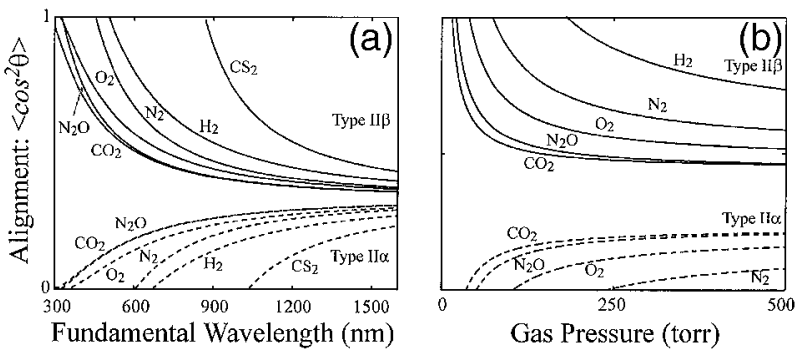

Fig. 2. Phase-matching curves for type II $\alpha$ (solid curves) and type II $\beta$ (dashed curves) THG processes for the molecular gases listed in Table 1. (a) Free-space alignment required for THG in molecular gases across a broad frequency range. (b) Gas pressure needed for THG with an $800-\mathrm{nm}$ fundamental beam in a $175-\mu \mathrm{m}$ hollow fiber.

pressures, the required alignment approaches that of free space because the dispersion of the waveguide becomes negligible compared with that of the gas.

Published dispersion curves $^{13,14}$ can be used to calculate the alignment required to phase match the THG process in an aligned molecular gas. Figure 2(a) shows the free-space alignment required for THG in molecular gases across a broad wavelength range; the dispersion of the critical alignment and the dispersion of the hollow-fiber balance make it possible to obtain extremely broad phase-matching bandwidths that can support few-cycle (i.e., $\ll 10-f s$ ) pulse durations in the vacuum ultraviolet. The lowest required alignment occurs for gases with a combination of low spectral dispersion between the fundamental and the harmonic fields and a relatively high intrinsic anisotropy. Both $\mathrm{CO}_{2}$ and $\mathrm{N}_{2} \mathrm{O}$ exhibit relatively low required alignment levels; $\mathrm{N}_{2} \mathrm{O}$ is easier to phase match due to its higher anisotropy. The alignment bandwidth, defined by the FWHM of the THG yield, is \pm 0.015 for a 10 -cm-long fiber with 100 Torr of $\mathrm{N}_{2} \mathrm{O}$. We also evaluated phase-matching conditions for phase matching at $800 \mathrm{~nm}$ for a number of linear molecules contained in a $175-\mu \mathrm{m}$-diameter hollow-core fiber. The results are summarized in Table 1 and plotted in Fig. 2(b). The minimum alignment pulse temporal duration required for sufficient alignment of the molecular gas to phase match THG frequency conversion in a hollow-core fiber is shown in Table 1 for a range of peak pulse intensity values. We estimate the conversion efficiency of the THG process to range from $1 \%$ to $20 \%$ for THG pulse durations of the order of $1.8-8 \mathrm{fs}$.

In summary, we have demonstrated that phasematched third-harmonic generation is possible in an ensemble of aligned linear molecules over a very broad wavelength range. One may also effectively combine this technique with time-dependent phase modulation $^{12}$ to obtain spectral broadening and subsequent compression, or it may be used with other nonlinear interactions. For example, a combination of transient birefringent and resonant phase matching may make
Table 1. Conditions Required for Phase Matching Third-Harmonic Frequency Conversion of 800-nm Light with Aligned Linear Molecules in a Hollow-Core Fiber for Peak Intensities (in femtoseconds) of $3 \times 10^{13}\left(\tau_{a}\right)$, $6 \times 10^{13}\left(\tau_{b}\right)$, and $9 \times 10^{13}\left(\tau_{c}\right) \mathrm{W} / \mathrm{cm}^{2}$

\begin{tabular}{lccccc}
\hline $\mathrm{Gas}$ & $K_{\alpha}$ & $K_{\beta}$ & $\tau_{a}$ & $\tau_{b}$ & $\tau_{c}$ \\
\hline $\mathrm{CO}_{2}$ & 0.2277 & 0.4420 & 162 & 72 & 48 \\
$\mathrm{~N}_{2} \mathrm{O}$ & 0.2257 & 0.4447 & 122 & 59 & 36 \\
$\mathrm{O}_{2}$ & 0.1995 & 0.4782 & - & - & 82 \\
\hline
\end{tabular}

it possible to tune resonant phase-matched frequencies over a larger range than previously possible. Secondharmonic generation in gases may also be possible in the case of polar molecules aligned with a half-cycle optical pulse. Finally, by control of the degree of alignment along the propagation direction, the nonlinear coefficient of the material can be periodically structured, allowing quasi-phase matching.

This work was supported by the Chemical Sciences, Geosciences and Biosciences Division of the Office of Basic Energy Sciences, U.S. Department of Energy, and by the National Science Foundation. We thank Chris H. Greene for useful discussions. R. A. Bartels's e-mail address is bartels@colorado.edu.

\section{References}

1. N. Bloembergen, Nonlinear Optics (World Scientific, Teaneck, N.J., 1996).

2. P. A. Franken, A. E. Hill, C. W. Peters, and G. Weinreich, Phys. Rev. Lett. 7, 118 (1961).

3. N. Katzenellenbogen and D. Grischkowsky, Appl. Phys. Lett. 58, 222 (1991).

4. M. Nisoli, S. D. Silvestri, V. Magni, and O. Svelto, Opt. Lett. 19, 1973 (1994).

5. A. McPherson, G. Gibson, H. Jara, U. Johann, T. S. Luk, I. A. McIntyre, K. Boyer, and C. K. Rhodes, J. Opt. Soc. Am. B 4, 595 (1987).

6. M. Ferray, A. L'Huillier, X. F. Li, L. A. Lompré, G. Mainfray, and C. Manus, J. Phys. B 21, L31 (1987).

7. A. Rundquist, C. G. Durfee III, S. Bakus, C. Herne, Z. Chang, M. M. Murnane, and H. C. Kapteyn, Science 280, 1412 (1998).

8. A. J. Merriam, S. J. Sharpe, M. Shverdin, D. Manuszak, G. Y. Yin, and S. E. Harris, Phys. Rev. Lett. 84, 5308 (2000).

9. A. H. Kung, J. F. Young, and S. E. Harris, Appl. Phys. Lett. 22, 301 (1973).

10. C. G. Durfee, S. Backus, M. M. Murnane, and H. C. Kapteyn, Opt. Lett. 22, 1565 (1997).

11. B. Friedrich and D. Herschbach, Phys. Rev. Lett. 74, 4623 (1995).

12. R. A. Bartels, T. C. Weinacht, N. Wagner, M. Baertchy, C. Greene, M. M. Murnane, and H. C. Kapteyn, Phys. Rev. Lett. 88, 019303 (2002).

13. U. Hohm, Chem. Phys. 179, 533 (1994).

14. D. Nikogasyan, Properties of Optical and Laser-Related Materials (Wiley, New York, 1997). 